The Be Phenomenon in Early-Type Stars, IAU Colloquium 175

ASP Conference Series, Vol. 214, 2000

M. A. Smith, H. F. Henrichs, and J. Fabregat, eds.

\title{
IUE Spectra of Be Star CX Dra: Evidence for Winds
}

\author{
Petr Škoda, Pavel Koubský \\ Astronomical Institute, AS CR, 25165 Ondřejov, Czech Republic
}

\begin{abstract}
The Be binary star CX Dra has been observed with the IUE satellite over a period of 14 years. More than $60 \mathrm{SWP}$ high resolution spectra were obtained. After processing the quotient flux spectra of the Al III and Si IV lines show very complicated behaviour. The most important features are the violet shifted components in these lines which show very negative velocities (up to $-800 \mathrm{~km} / \mathrm{s}$ ) in a particular phase interval.
\end{abstract}

\section{Introduction}

The binary CX Dra (HD $174237=$ HR $7084=$ MWC608, B2.5Ve, $V=5.9$, $v \sin i=160 \mathrm{~km} \mathrm{~s}^{-1}$ ) has been long known as an emission-line star (Mohler, 1940). Lacoarret (1965) found cyclic variations in the $\mathrm{H} \alpha$ emission with a characteristic time scale of 5 to 7 days. Merlin (1975) discovered light variations of the star with a similar time scale of 7 to 12 days. Koubský $(1976,1978)$ analysed the radial velocities of the He I photospheric lines and showed convincingly that CX Dra is a 6.696-day spectroscopic binary $(K=35 \mathrm{~km} / \mathrm{s})$. The fact that the emission in the $\mathrm{H} \alpha$ line varies on a time scale similar to the orbital period led to the conclusion that CX Dra is an interacting binary.

The photometric study of CX Dra by Koubský et al. (1980) revealed longterm irregular fluctuations up to $0.2 \mathrm{mag}$ in $V$, and lower amplitude residual variations phase-locked with the spectroscopic period. Guinan et al. (1984) detected CX Dra as a moderately strong X-ray source with the Einstein Observatory. They suggested that the X-rays originated from the corona of the secondary component. This interpretation was also in good agreement with their analysis of the residual light curve detected by Koubský et al. (1980). Guinan et al. (1984) have shown that the light curve conforms with the presence of an early-type primary $\left(T_{\mathrm{e}}=20000 \mathrm{~K}\right)$ and a later-type companion $\left(T_{\mathrm{e}}=6500 \mathrm{~K}\right)$ in the system.

The lines of the secondary component were detected in 1985 on Reticon spectra taken with the Shane Telescope at the Lick Observatory by G.D. Penrod. Horn et al. (1992) derived the orbital parameters for both components based on 17 electronic spectra secured during one orbital cycle with the Aurélie spectrograph at the Haute Provence Observatory. They concluded that the orbital inclination of the system is in the range $i=52^{\circ}-55^{\circ}$. The resulting mass ratio of the components $q$ was found to be close to 0.24 . The spectral type of the secondary was estimated to be F5 III. 

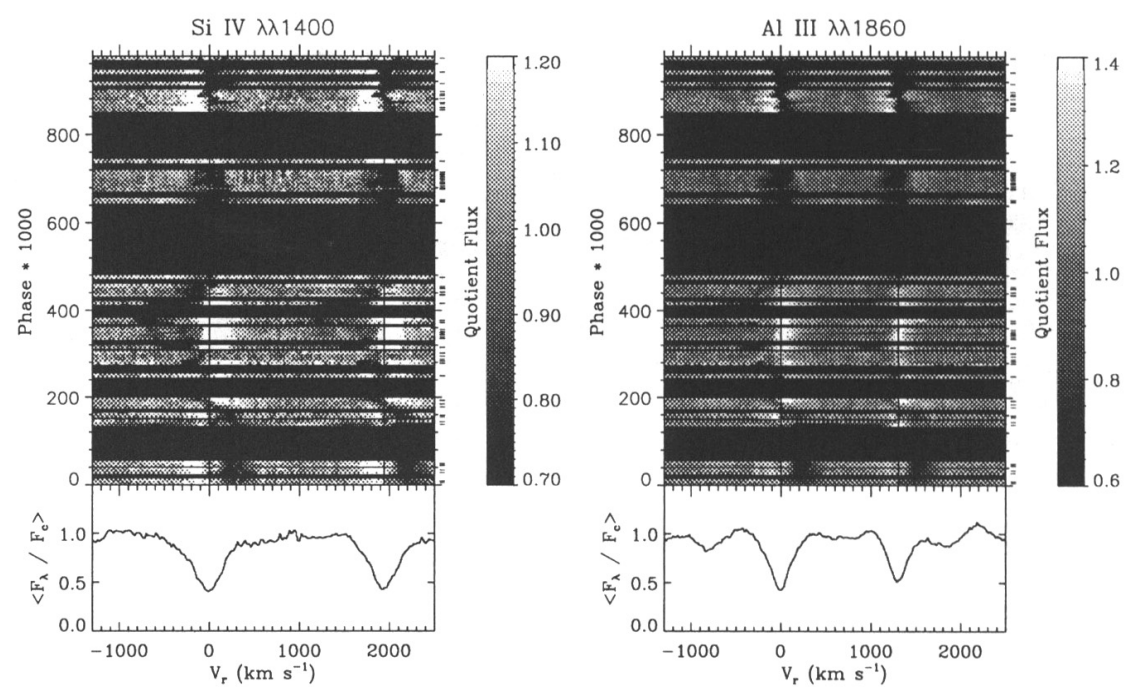

Figure 1. Dynamic spectra of CX Dra for the Si IV and AlIII lines

\section{The observations}

CX Dra was extensively observed with the IUE satellite. Since 1978 more than ninety high resolution UV spectra were secured during the lifetime of the satellite (including 64 SWP images). Peters and Polidan (1984) reported strong variable $\mathrm{N}$ V, C IV and Si IV lines.

In the present study we used the MXHI files of all available SWP spectra of CX Draconis from the IUE Final Archive (ripple corrected net flux data). The processing was performed with the help of the IDL package set, kindly provided to us by Derck Massa from NASA GSFC. In this process each spectrum was normalized by the mean of all spectra. This way we obtained quotient flux spectra which were then used for further analysis.

In Figure 1 we present dynamic spectra of CX Dra obtained during the life-time of the IUE satellite folded with the 6.7 days orbital phase (ephemeris by Richards et al. 1999). The two spectral regions are centered on Si IV $1393 \AA$ (left) and Al III $1854 \AA$ (right). The mean spectrum is displayed in the lower part of each panel. The short ticks on right side of each panel represent the time of exposure in the phase space.

In order to better illustrate the behaviour of these lines during the interesting phases $0.25-0.48$ we present them in Figure 2 as stacked over-plots spread in phase. 

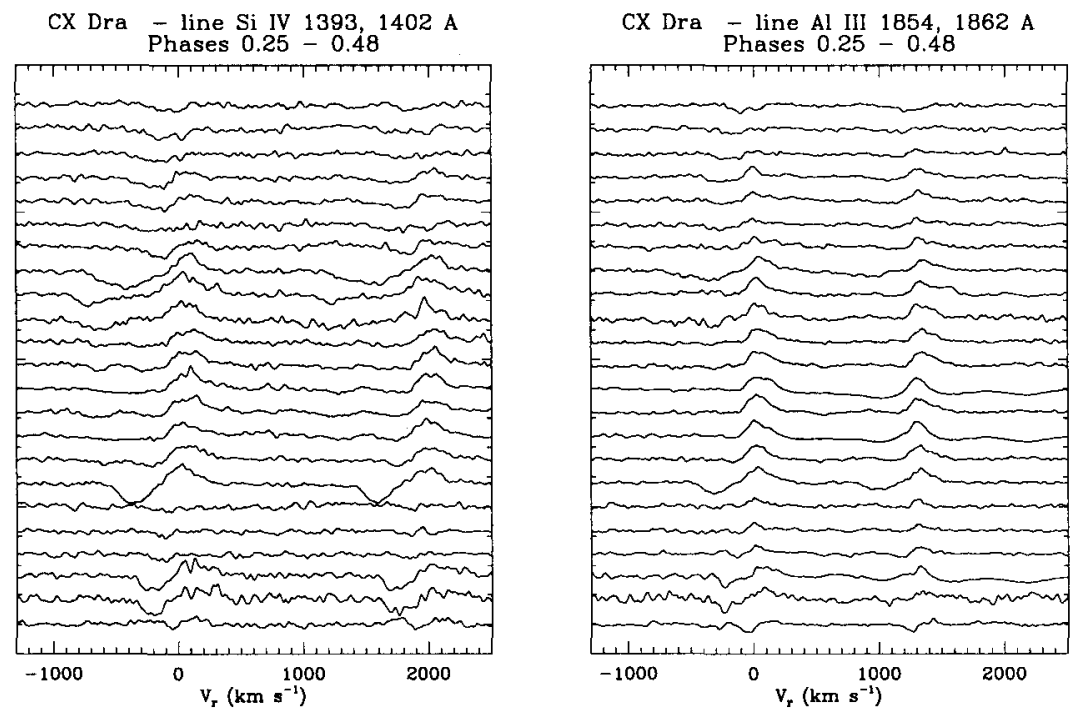

Figure 2. Quotient flux spectra of Si IV and AlIII lines in the phase interval $0.25-0.48$

\section{Discussion and Conclusions}

The most interesting feature in these figures is the appearance of violet-shifted satellite components in the phase interval $0.25-0.45$. Wonnacott (1988) interpreted these observations as due to a jet of material which escapes from the system. Our dynamic spectra confirms that this feature is variable on the longterm time scale. Note also that a "quasi-emission" appears near zero velocity at the time when the violet satellite is visible. The other interesting feature is a red shifted absorption in the vicinity of phase 0.0, thus giving a hint that the spectrum of the circumstellar medium in CX Dra mimics an asymmetric "S wave".

Acknowledgments. We are grateful to Derck Massa for sending us his version of IDL package used for the reduction of IUE data. The original MXHI files were obtained from the IUE Final Archive at STScI.

This work was partially supported with the subvention by the Ministry of Education, Youth and Sports of the Czech Republic (grants LB98251, ES014) and NSF grant INT-9512791.

\section{References}

Horn, J., Hubert, A.M., Hubert, H., Koubský, P., Bailloux, N. 1992, A\&A 259, L5

Guinan, E.F., Koch, R.H., Plavec, M.J. 1984, ApJ 282, 667

Koubský, P. 1976, IAU Inf. Bull. Var. Stars 1188 
Koubský, P. 1978, Bull. Astron. Inst. Czechosl. 29, 288

Koubský, P., Harmanec, P., Horn, J., Jerzykiewicz, M., Kříž, S., Papoušek, J., Pavlovski, K., Ždárský, F. 1980, Bull. Astron. Inst. Czech. 31, 75

Lacoarret, M. 1965, Ann. Astrophys. 23, 231

Merlin, P. 1975, A\&A 39, 139

Mohler, O. 1940, ApJ 92, 315

Peters, G.J., Polidan, R.S. 1984, ApJ 283, 745

Richards, M.T., Koubský, P., Šimon, V., Peters, G. J., Hirata, R., Škoda, P., Masuda, S. 1999, ApJ, accepted

Wonnacott, D. 1988, in Decade of UV Astronomy with IUE Satellite, Ed. R.J. Rolfe, ESA SP 281, Vol. 1, 209 